\title{
Building interior layout reconstruction from through-the-wall radar image using MST-based method
}

\author{
Bo Chen, Tian Jin*, Biying Lu and Zhimin Zhou
}

\begin{abstract}
In order to reconstruct the building interior layout from the through-the-wall radar image, this paper proposes a method based on minimum spanning tree (MST) which comes from the graph theory. The building layout graph (BLG) model is firstly established using the wall-wall-floor trihedrals inside the building. The vertex set and the edge set including the weight between any two nodes of the graph are defined afterwards. Then, the MST-based method is used to reconstruct the building interior layout. We have also improved the traditional method by applying the MST model with restricted conditions to solve the reconstruction problem when there exist interference targets in a building. The anechoic chamber measurements are carried out to demonstrate the suitability and efficacy of the MST-based method.
\end{abstract}

Keywords: Through-the-wall radar image (TWRI); Minimum spanning tree (MST); Building layout graph (BLG); Graph theory

\section{Introduction}

Sensing through walls is an emerging technology in recent years. Through-the-wall radar imaging (TWRI) is considered to be very effective to achieve the objectives of 'seeing' through walls using electromagnetic waves. TWRI is highly desirable for a range of organizations, including police, rescue personnel missions, surveillance, first responders, and defense forces [1,2]. Most throughthe-wall radars in use often require a close position to the wall or they have to be pressed against the wall. Moreover, TWRI mainly focuses on behind-the-wall target. These systems only have the single-wall penetrating ability, providing range, cross-range, and motion information of behind-the-wall targets [3-5].

In recent years, some research institutes in America and Europe are focusing on through-the-wall radar systems which have the ability of reconstructing the interior layout of a building. These systems will greatly improve security, response time, covertness, and reliability of the firefighting and law enforcement tasks. Typical systems include the SAPPHIRE system designed

\footnotetext{
* Correspondence: tianjin@nudt.edu.cn

College of Electronic Science and Engineering, National University of Defense Technology, Changsha, Hunan 410073, China
}

by the Netherlands Organization for Applied Scientific (TNO) and the Visibuilding program sponsored by the Defense Advanced Research Projects Agency (DARPA). The operating principle of SAPPHIRE is detecting and identifying the principal scatterers inside a building, and then deducing the interior layout [3,4]. Visibuilding program uses model-based signal processing method to reconstruct the building layout. In addition, the Army Research Laboratory (ARL) had conducted several field experiments to image an abandoned barrack. During the experiment, the radar system moves along two mutual vertical sides and the two radar images are synthesized coherently to reconstruct the interior layout $[6,7]$.

For specific building interior layout reconstruction methods in literatures, they are divided into two types: the first one is using multi-look fusion approach to through-the-wall radar images from multi-view measurements, such as the experimental system of ARL mentioned above; the other one is analyzing through-the-wall radar image and extracting the principal scatterers inside the building, namely, obtaining the position and orientation from region of interest (ROI) of the wall-wall-floor trihedral, then using reasonable deducing method to infer the interior layout, such as SAPPHIRE system. Comparing 
with the first type, the advantage of the second type lies in using the electromagnetic scattering characteristics sufficiently, keeping away from the influence of clutter and multi-path propagation and ensuring robustness [8].

In our previous work [9], a method of acquiring the attributes of a trihedral is presented. The orientation information of all the trihedrals in a through-the-wall radar image can be obtained by a virtual aperture imaging model [10]. In addition, the estimation accuracy satisfies the requirements of reconstructing the building interior layout. Therefore, this paper mainly focuses on designing the reconstruction method. If the position and orientation information of all the trihedrals appeared in through-the-wall radar image are known, the whole building interior layout can be obtained immediately by direct artificial judgment on one-time $[3,11]$. However, they are based on simulation data only and lack high automation.

The problem of the one-time artificial judgment is that the reconstruction accuracy depends greatly on the signal-to-noise ratio (SNR) of through-the-wall radar image. Once there exist interference targets or undetected wall-wall-floor trihedrals, the reconstructed layout will be different from the true layout. Therefore, some researchers propose an iterative reconstruction method based on electromagnetic scattering model $[8,12]$. This method is formed by prediction stage and test stage. The prediction stage in the iterative procedure is the same as one-time reconstruction process. The predicted radar image can be obtained according to the predicted layout using electromagnetic modeling method. During the test stage, the measured radar image and the predicted radar image should be compared to modify the predicted building model, making a more accurate description of the building. Each iteration will add absent walls or cut out extra walls so as to be closer to the true layout of the building, comparing with the pre-iteration process. Then the positions of the wall-wall-floor trihedrals will be more accurate after compensation of the wall parameters [13]. To improve the computation efficiency of the iterative reconstruction method, prevent manual intervention during the reconstruction process and realize online computation, an effective building interior layout auto-reconstruction method need to be investigated.

Without loss of generality, this paper focuses on common building designs with regularity and rectilinearity. The interior walls are either perpendicular or parallel to the exterior walls. In addition, any closed structure is not allowed to be existed if the exterior walls of the building are eliminated. The graph-based reconstruction method proposed in this paper comes from recent papers on the subject of floor plan estimation $[14,15]$. They also employ graph theory in its problem formulation. This paper proposes a graph-theoretical building interior layout reconstruction method using the prototype of their method. However, the way that graphs are employed in this paper is different: the edges of the graph are used to indicate the walls that are present or absent, and the nodes are used to represent the wallwall-floor trihedrals inside the building.

This paper is organized as follows: Section 2 gives a brief description of the experimental system in the anechoic chamber. Section 3 develops a graph-theoretical model for a rectangular floor plan which is called building layout graph (BLG). After the graph elements and the weight of each edge are defined, the minimum spanning tree (MST) method is used to reconstruct an experimental building using the radar system mentioned in Section 2. Section 4 probes into how to improve the MST-based reconstruction method when there exist interference targets in the building and also presents the experimental results. Section 5 gives some remarkable conclusions.

\section{Overview of the experimental system}

Before we introduce the MST-based method, we will describe the experimental system at first in this section. The proposed MST-based method is validated by the real data collected in an anechoic chamber, as is shown in Figure 1. The experimental system is composed of a vector network analyzer (VNA) and two horn antennas. The horn antennas fixed on the position system move from left to right, forming an aperture with $3.8 \mathrm{~m}$ in length. The azimuth interval is $0.05 \mathrm{~m}$ and the system covers the frequency band from 2 to $4 \mathrm{GHz}$ with a step of $2 \mathrm{MHz}$.

\section{MST-based reconstruction method for building layout}

The building interior layout reconstruction algorithm presented in this paper is based on MST which belongs to the graph theory. In this section, we will define the elements in BLG and establish the relationship between the building interior layout reconstruction problem and the MST.

\subsection{Elements in BLG and MST}

Actually, if the walls are treated as the connected lines between a couple of nodes, the building interior layout can be treated as a weighted undirected graph. This graph will be named as BLG for short in the following text. A graph is usually denoted as $G(V, E)$, where $V$ is the node set (or vertex set) and $E$ is the weighted edge set which connects all the nodes. The node set, edge set, and other nomenclatures are defined as follows:

(i) Nodes (or Vertices): The wall-wall-floor trihedrals inside the building are treated as nodes of the building layout graph. 


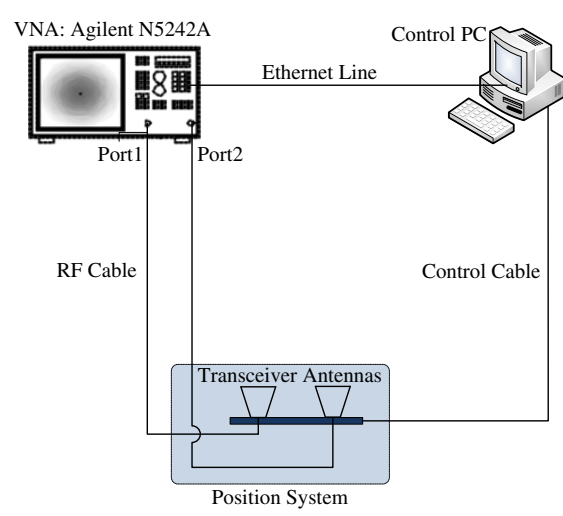

(a)

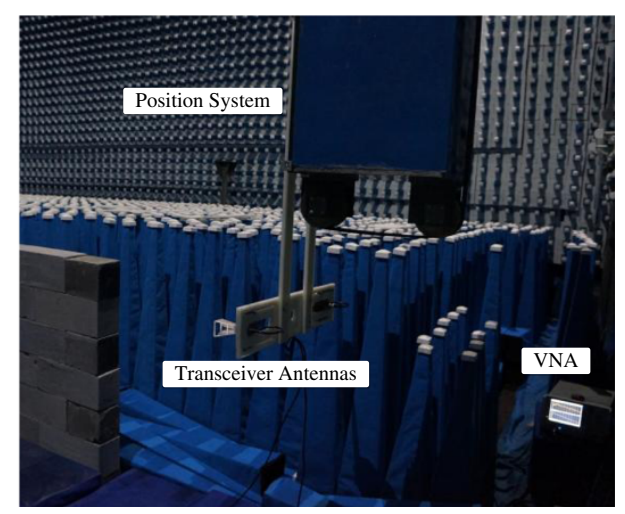

(b)

Figure 1 Radar measurement setup in the anechoic chamber. (a) Experimental radar setup. (b) Optical photo.

(ii) Edges: Walls existing between two adjacent nodes, all the edges in BLG are undirected.

(iii) Degree: It is used to describe a node. It means the number of walls which relates to the current wallwall-floor trihedral node. For undirected graph, in-degree and out-degree are both treated as uniform degree with no difference. The degree of node $v$ is denoted as $D(v)$.

(iv) Order: It is used to describe a graph. It means the number of trihedral nodes in the building graph and is denoted as $|V|$.

Positions and orientations of the wall-wall-floor trihedrals are attributes of the node set. A weighted undirected graph can be developed if we have got all the trihedrals including their attributes through radar measurements. How to acquire these attributes has been researched. From [9], we have known that the pose angles of all the trihedrals in a radar image can be estimated by using a virtual aperture imaging model. It will not be discussed in this paper to avoid unnecessary repetition.

If a graph with any pair of nodes being connected by at least one edge, it is called connected graph. In a complete graph, any two nodes is connected by an edge. Therefore, a weighted complete undirected graph must be a connected graph. Given a weighted complete undirected graph $G(V, E)$ with $|V|=N$, the number of trees (a subgraph of $G$ without closed loops) that connects all the nodes of the graph is $N^{N-2}$. The MST is the tree with the minimum total weight, defined as the sum of the weight of each tree's edge.

As is mentioned afore, in a radar measurement data set consisting of reflected signals from principal scatterers, we consider the wall-wall-floor trihedrals as the nodes of a graph, the horizontal or vertical wall lines being the edges joining the nodes. Any possible layout predicted according to present nodes corresponds a spanning tree of the weighted complete undirected graph BLG. If we have defined reasonable edge weight, we could make the total weight of the spanning tree corresponding to the correct interior layout to be minimum. By this way, we can translate the building interior layout reconstruction process into searching the MST of the complete weighted undirected graph. Therefore, how to construct the edge weight becomes the key of the reconstruction process.

\subsection{Definition of edge weight in BLG}

The purpose of defining edge weight is making equivalence between the correct building interior layout and the MST in BLG. Before we define the edge weight, we will give the definition of angle range of each trihedral node in advance. Figure 2 gives the angle range definition when the degree of a trihedral node equals 2,3 , and $4 . \theta_{s}$ means the starting angle and $\theta_{e}$ means the ending angle. The angle range is denoted as $\left[\theta_{s}, \theta_{e}\right]$. Due to the rectangular shape of the building, the orientation of trihedrals is divided into four quadrants.

Next, we will define the edge weight between a couple of trihedral nodes. To make correspondence between correct building interior layout and the MST in BLG,

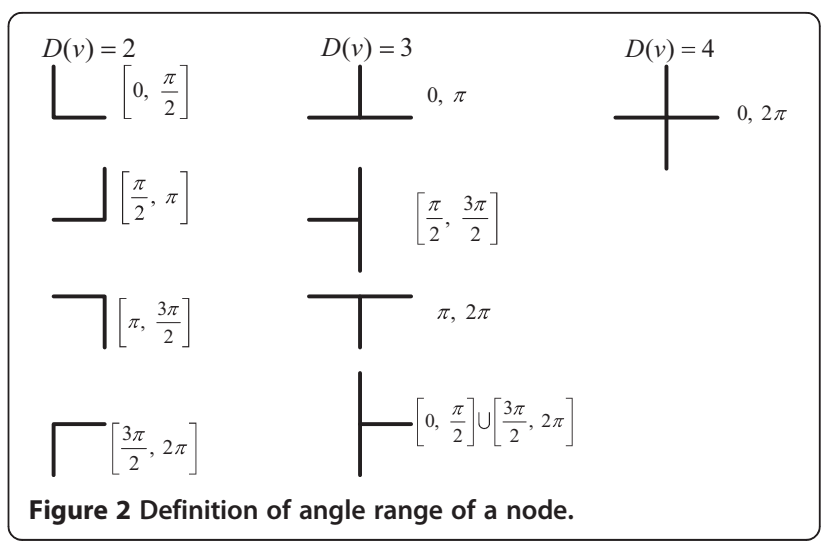


the definition of the edge weight $d(i, j)$ between a couple of nodes which are denoted as $v_{i}$ and $v_{j}$ should subject to the following four conditions:

(i) Due to the rectangular shape of the building, if an edge connecting two nodes is closer to the horizontal or vertical direction than another edge, the weight of this edge should be smaller. In contrast, if an edge is farther from the node angle range which is defined above, the weight should be larger.

(ii) The edge weight should monotonically increase with its Euclid distance. Namely, one of two edges whose Euclid distance is smaller should also be smaller in weight if they have the same node angles.

(iii) If the edge weight is defined as an exponential form, the base number should not be equal to 1 , and the power exponent should not be equal to 0 . Otherwise, the weight cannot be distinguished from angle range and Euclid distance.

(iv) Considering that the positions of the nodes may be departed from their true positions and the position errors will influence the reconstructed interior layout, the edge weight should have a certain faulttolerant ability for this error. In other words, the edge weight function should be continuous at the boundary of the angle range. It is not allowed to have a sudden jump in value at the starting angle $\theta_{s}$ and the ending angle $\theta_{e}$.

From the above four conditions, we could see that the edge weight between a couple of nodes should consider not only their Euclid distance but also the angle constraint. If we have constructed an edge weight that is satisfying the above four conditions, the building interior layout reconstruction process can be translated into searching the MST of BLG. The edge weight for the nodes $v_{i}$ and $v_{j}$ is defined in
(1) with $\left(x_{i}, y_{i}\right)$ and $\left(x_{j}, y_{j}\right)$ representing their position attributes, respectively.

$$
d(i, j)=\left(\frac{l_{i j}}{L}\right)^{\gamma\left(\theta_{i}, \theta_{j}\right)}
$$

where $l_{i j}$ is the Euclid distance between node $v_{i}$ and node $v_{j}$ :

$$
l_{i j}=\sqrt{\left(x_{i}-x_{j}\right)^{2}+\left(y_{i}-y_{j}\right)^{2}}
$$

$\gamma\left(\theta_{i}, \theta_{j}\right)$ is the exponential term of the edge weight, where $\theta_{i}$ and $\theta_{j}$ represents the angle of the edge $e_{i j}=\left(v_{i}, v_{j}\right)$ which connects nodes $v_{i}$ and $v_{j}$. The definition of $\theta_{i}$ and $\theta_{j}$ is shown in Figure 3. $L$ represents the perimeter of the building outline which is known in advance. The introduction of $L$ is to keep the base number from equaling to 1 , matching the third condition. Otherwise, the edge weights will have no difference for Euclid distance.

Considering the geometry relationship between any two nodes shown in Figure 3, the node angle is defined as follows:

$$
\left\{\begin{array}{l}
\theta_{i}=\pi+\operatorname{sgn}\left(y_{i}-y_{j}\right) * \arccos \frac{x_{i}-x_{j}}{l_{i j}} \\
\theta_{j}=\theta_{i}-\operatorname{sgn}\left(y_{i}-y_{j}\right) * \pi
\end{array},\right.
$$

where $\operatorname{sgn}(y)=\left\{\begin{array}{ll}1, & y \geq 0 \\ -1 & y<0\end{array}\right.$.

In the edge weight definition, we will make some analysis on the exponential form $a^{x}(0<a<1)$. If $0<a_{2}<a_{1}<1$ and power exponent $x>0, a_{2}^{x}<a_{1}^{x}$ when $x$ is fixed. This feature matches the monotonicity between the Euclid distance and the edge weight. Moreover, $a^{x}(0<a<1)$ is a monotonically decreasing function with $x$, in order to keep equivalence between the MST and the correct layout, the power exponent $\gamma\left(\theta_{i}, \theta_{j}\right)$ should get the maximum value when $\theta_{i}$ or $\theta_{j}$ equals $0, \pi / 2, \pi, 3 \pi / 2$,

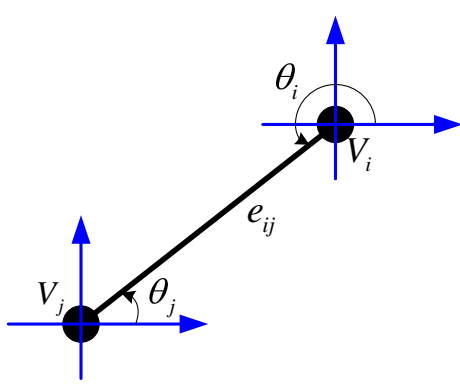

(a)

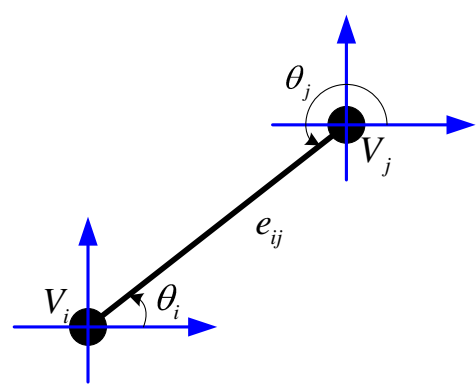

(b)

Figure 3 Definition of node angle. (a) Case 1: $y_{i}>y_{j}$ (b) Case 2: $y_{i}<y_{j}$. 
and $2 \pi$, making the edge weight between these two nodes get the minimum value. As a result, trigonometric function is the basic form of the power exponent $\gamma\left(\theta_{i}, \theta_{j}\right)$ we considered, satisfying the first condition.

According to the above description and conditions, the exponential term of the edge weight $d(i, j)$ in the node angle range can be expressed as follows:

$$
\gamma(\theta)=-|\sin (2 \theta)|+\beta \quad\left(\theta \in\left[\theta_{s}, \theta_{e}\right], \beta>1\right)
$$

and

$$
\gamma\left(\theta_{i}, \theta_{j}\right)=\gamma\left(\theta_{i}\right)+\gamma\left(\theta_{j}\right)
$$

There is a variable $\beta$ in the definition of power exponent $\gamma\left(\theta_{i}, \theta_{j}\right) . \beta$ is introduced in order to keep the exponential term above zero. Thus, $d(i, j)$ will increase with the Euclid distance when $\theta_{i}$ and $\theta_{j}$ are within the angle range. This feature matches the second condition.

In addition, edge weight should also keep symmetry out of the angle range, satisfying the fourth condition. Combining with (4), the power exponent definition shown in Figure 4 is considered. We take the starting angle $\theta_{s}=\pi / 2$ and the ending angle $\theta_{e}=3 \pi / 2$ as an example. The power exponent is in trigonometric form within the node angle range and in parabolic form out of the angle range. It will be similar when angle range adopts other values.

\subsection{MST searching method}

We have given the definition of edge weight of the BLG and made equivalence between the correct building interior layout and the MST. Now we should focus on how to find the MST of the building graph so as to obtain the interior layout of the building.

The frequently used MST searching algorithms are the Kruskal algorithm and the Prim algorithm [16-18]. Both of these two algorithms come from greedy ideas. However, proof has been shown that these algorithms would obtain the global optimization results instead of being

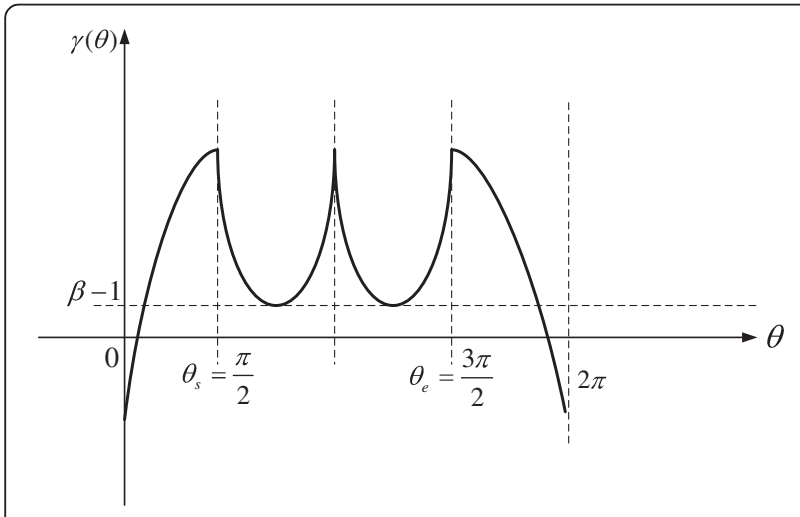

Figure 4 Power exponent of the edge weight definition. driven to the local optimization results. The Kruskal method needs to sort the edge weight only one time, while the Prim method needs to sort the edge weight more than one time. As a result, we have used the algorithm of Kruskal to construct our MST for computation efficiency. The process of the Kruskal algorithm in building interior layout reconstruction is described with four steps:

Step 1: Using definition in (1) (5), sort the edge weight of the weighted undirected building graph in ascending order;

Step 2: Set $i=1$ and let the initial edge be $E_{0}=\varnothing$; Step 3: Select an edge $e_{i}$ of minimum weight value not in $E_{i-1}$ such that $T_{i}=<E_{i-1} \cup\left\{e_{i}\right\}>$ is acyclic and define $E_{i}=E_{i-1} \cup e_{i}$. If no such edge exists, let $T=<E_{i}>$ and stop;

Step 4: Replace $i+1$ by $i$. Return to step 3 .

After $N-1$ iterations, where $N$ is the order of the building layout graph, the complete MST is found. Thus, the building interior layout is obtained subsequently.

\subsection{Experimental results}

In the above sections, we have translated the building layout reconstruction problem into the MST searching problem. With the edge weight defined as (1) (5) and the Kruskal algorithm, some simulations have been implemented and some valuable results are obtained. We have known that after the outmost trihedrals located on the outline which are called priori nodes are removed, the MST of the graph formed by the remaining nodes represents the interior layout of the building [19].

To validate the above MST method in real radar measurement data, we have constructed some experiments in the anechoic chamber using the radar system depicted in section 2. Figure $5 \mathrm{a}$ gives the photograph of the radar imaging scene. Figure $5 \mathrm{~b}$ is the back projection (BP) result of the radar echo acquired by the VNA.

The ROI of the principal scatterers can be acquired by using constant false alarm rate (CFAR) detection method and morphology operation to the building radar image shown in Figure 5b. Hereto, we have obtained all the nodes in BLG including their position attributes. Due to the rectangular characteristics of the building, the orientation of the wall-wall-floor trihedral has definite four types. As a result, the orientation attribute of the trihedral can be determined as soon as we have gotten their position attributes. Figure 6 is the MST result using the Kruskal method based on the extracted nodes from radar image as well as attribute information except priori nodes. Different colors represent different orientations of the node.

The result in Figure 6 represents the correct interior layout of the building in the anechoic chamber. Combining 


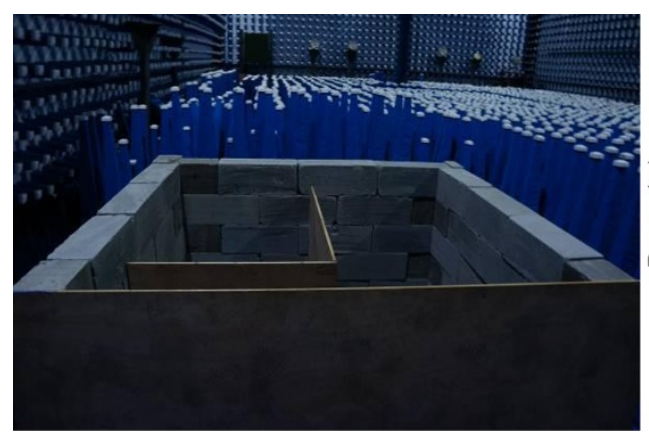

(a)

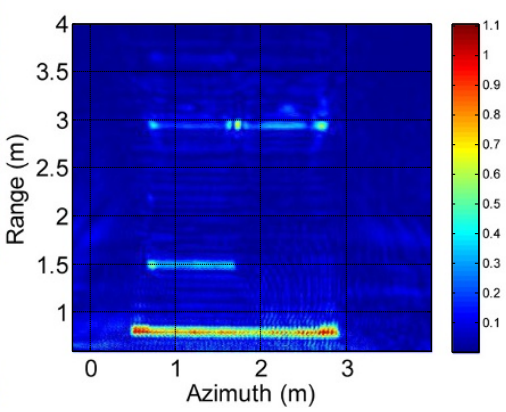

(b)

Figure 5 The anechoic chamber radar image. (a) Photograph of the imaging scene. (b) Radar image.

the priori outline information (shown by the dashed line), the whole layout of the building can be obtained.

\section{Reconstruction of building layout with interferences}

\subsection{Drawback of the traditional MST-based method}

In practical radar measurements, there will usually be targets which are not principal scatterers such as human targets inside the building. These targets tend to bring about some interference target appearance in the radar image which do not belong to wall-wall-floor trihedral, resulting in some interference nodes in BLG and affecting the final reconstructed interior layout subsequently. Figure 7 presents the photograph of the anechoic chamber measurement scene with interference targets and the back projection (BP) imaging results of the radar return. Two metal cylinders are placed in the imaging area to simulate the interference targets inside the building.

The following figure is the reconstructed result using the classical Kruskal algorithm based on all the nodes except those priori nodes and their corresponding orientation

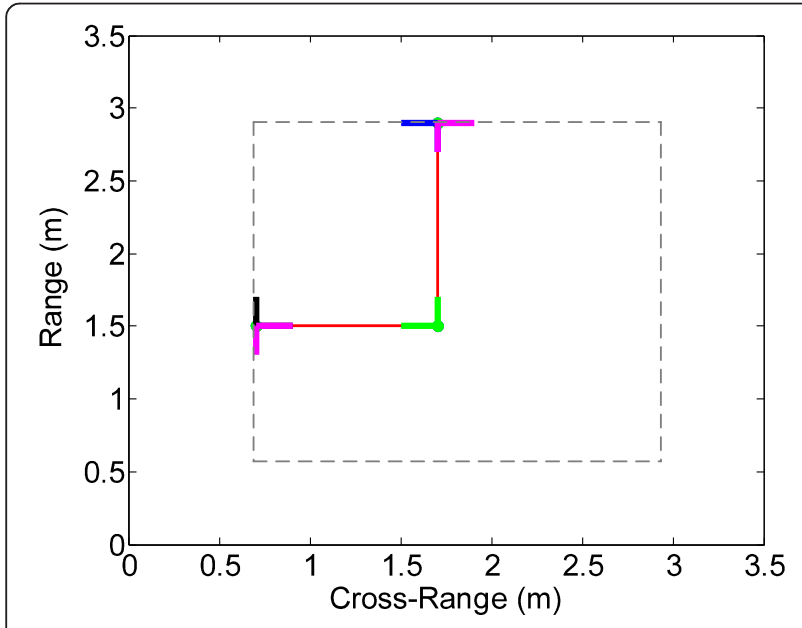

Figure 6 MST of the anechoic chamber building. information. The orientation of the cylinder is considered to be omnidirectional, namely covers all the four quadrants. Figure $8 \mathrm{a}$ gives the nodes distribution in this case and they are denoted from 1 to 5 . The generated MST is shown in Figure $8 \mathrm{~b}$.

\subsection{Improved MST-based method}

It is clear that the generated MST is different with the correct building interior layout when the interference targets exist. As a result, we need to make some improvements of the traditional MST-based method. Considering the rectangular-shape assumption of the building, the interior layout reconstruction procedure with interference nodes is improved as follows:

Step 1: Extract the trihedral nodes and dihedral edges information in the radar image;

Step 2: Search the MST with restricted conditions based on the nodes and edges information, denote the restricted MST as $T_{1}$;

Step 3: Traverse every nodes of $T_{1}$ and calculate the node angle;

Step 4: Pick out the nodes whose angle is not divisible by $\pi / 2$;

Step 5: Apply Kruskal algorithm to the remaining nodes. The generated MST $T_{2}$ represents the final structure of the building.

Since the method of acquiring the principal scatterers such as dihedral and trihedral has been given in [8], how to extract the nodes and edges information of the BLG will not be discussed in this paper. We will focus on how to apply the MST-based method in building reconstruction when there exist some interference targets. From the procedure shown above, the key to this problem is how to solve the MST with restricted conditions [20]. The restricted conditions are the nodes and edges information obtained from the building radar image. An edge extracted from the radar image means that the two 


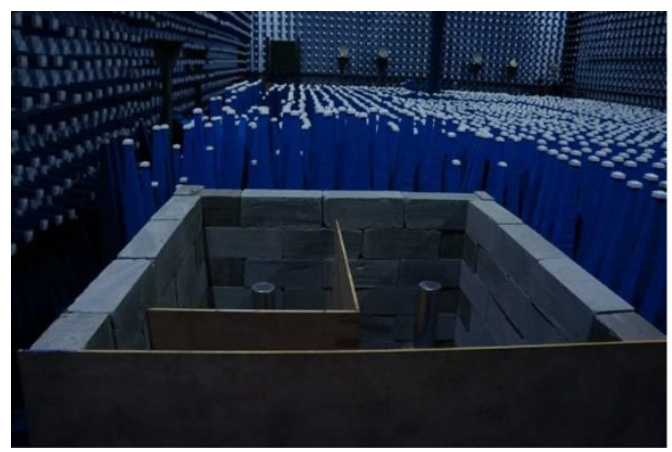

(a)

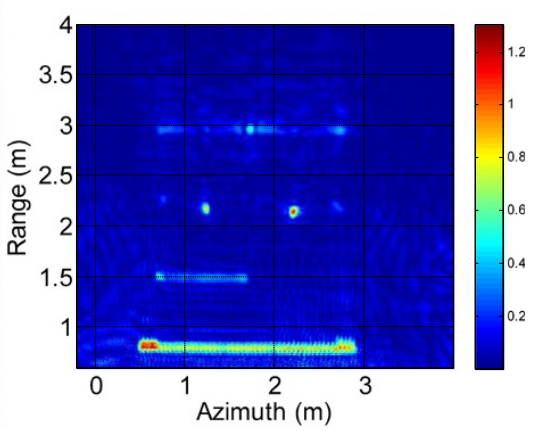

(b)

Figure 7 Radar image with interference targets. (a) Photograph of the imaging scene. (b) Radar image.

nodes which are related to this edge should be connected in the final generated MST definitely. This problem is described as follows using mathematical language.

Assume $G(V, E)$ is a weighted undirected connected graph, $G_{1}\left(V_{1}, E_{1}\right), \ldots, G_{r}\left(V_{n} E_{r}\right)$ are the $r$ subtrees which do not intersect with each other. If a spanning tree $T=\left(V, E^{\prime}\right)$ of the graph $G$ contains all the existed subtree, namely, $E_{i} \subset E^{i}(i=1,2, \ldots, r)$, moreover, the sum of all the edge weight of $T$ is the minimum, then $T$ is called the MST of $G$ with restricted conditions $G_{1}, \ldots, G_{r}$.

For convenience, the $r$ subtrees are merged into a forest $T_{0}=\left(V_{1} \cup \ldots \cup V_{n}, E_{1} \cup \ldots \cup E_{r}\right), d(u, v)$ represents the weight of the edge $(u, v)$ connecting node $u$ and node $v$. The following text gives the detailed construction method of MST with restricted conditions. The method is divided into two steps.

Step 1: Assume $U_{0}=V_{1} \cup \ldots \cup V_{r}, B_{0}=E_{1} \cup \ldots \cup E_{r}$, $T_{0}=\left(U_{0}, B_{0}\right)$ and $W_{0}=V-U_{0}$, take iteration in the following way:

$$
d\left(u_{0}, w_{0}\right)=\min _{\substack{u \in U_{k} \\ w \in W_{k}}} d(u, w)
$$

Let $U_{k+1}=U_{k} \cup\left\{w_{0}\right\}, W_{k+1}=W_{k}-\left\{w_{0}\right\}, B_{k+1}=$ $B_{k} \cup\left\{\left(u_{0}, w_{0}\right)\right\}$ and $T_{k+1}=\left(U_{k+1}, B_{k+1}\right)$, keep iteration until $W_{n}=0$. The forest characteristics will not be broken in the above iteration process due to the link of only one edge when adding nodes to $U_{k}$ each time. Moreover, we have not carried connection inside the forest, so the number of trees in the forest keeps unchanged. When the recursion stops, $T_{n}=\left(U_{n}, B_{n}\right)$ will be a forest covering all the nodes in $G$. The original $G_{i}$ will be expanded into $G_{i}^{\prime}\left(V_{i}^{\prime}, E_{i}^{\prime}\right)$

$(i=1,2, \ldots, r)$.

Step 2: Add some appropriate edges to $T_{n}$ to make it a spanning tree of $G$. Denote $U_{0}^{\prime}=V_{1}^{\prime}, W_{0}^{\prime}=V$ $U_{0}^{\prime}, B_{0}^{\prime}=E_{1}^{\prime} \cup \ldots \cup E_{r}^{\prime}$, if $U_{k}^{\prime}, W_{k}^{\prime}$, and $B_{k}^{\prime}$ have been constructed, there exist $w_{0}$ in $V_{j}^{\prime}$ and $u_{0}$ in $U_{k}^{\prime}$ which are subject to

$$
d\left(u_{0}, w_{0}\right)=\min _{\substack{u \in u_{k}^{\prime} \\ w \in W_{k}^{\prime}}} d(u, w)
$$

Let $U_{k+1}=U_{k}^{\prime} \cup V_{j}^{\prime}, W_{k+1}=V-U_{k+1}^{\prime}$, and $B_{k+1}^{\prime}=B_{k}^{\prime} \cup$ $\left\{\left(u_{0}, w_{0}\right)\right\}$, keep iteration until $W_{r}=0$. Each

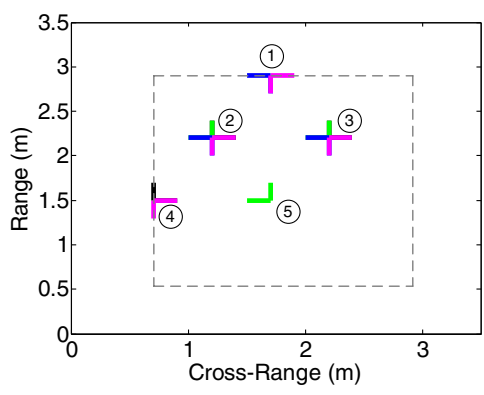

(a)

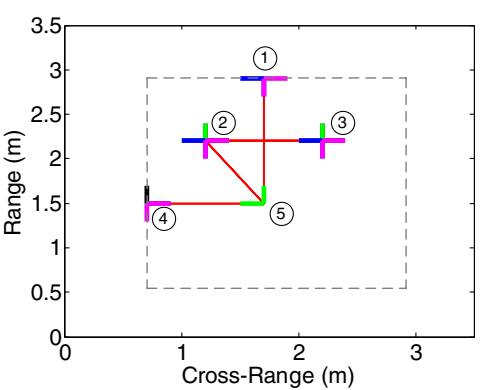

(b)

Figure 8 MST with interference targets. (a) Node distribution. (b) MST generated by the Kruskal algorithm. 


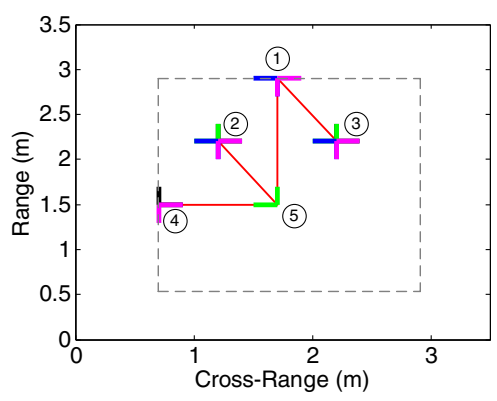

(a)

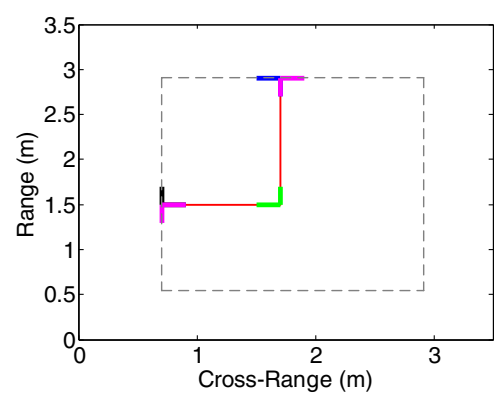

(b)

Figure 9 MST generated by improved method. (a) MST with restricted conditions. (b) MST with interference targets being removed.

iteration will add one edge which connects two isolated tree. The spanning tree $T=\left(V, B_{r-1}^{\prime}\right)$ of $G$ will be available when the iteration stops.

\subsection{Experimental results with interferences}

In [20], it has been proved that the spanning tree $T$ obtained by the above two steps is the MST satisfying the restricted conditions. For the radar image shown in Figure $7 \mathrm{~b}$, it is clear that the final MST should contain the edge formed by node 4 and node 5 according to the extracted edge information. Moreover, the final MST should not contain the edge formed by node 2 and node 3 since the edge does not appear in the radar image. In order to apply the improved method, the weight between node 2 and node 3 is assumed to be infinite. Figure 9a gives the MST generated by the above method. It is denoted as $T_{1}$.

After we have gotten $T_{1}$, the angle attribute of each node can be calculated according to the definition in section 3.2. Those nodes which do not satisfy the horizontal or perpendicular conditions are picked out. Assume the degree of node $v_{i}$ is $p$, its angle attribute is denoted as $\left(\theta_{i 1}, \theta_{i 2}, \ldots, \theta_{i p}\right)$. If

$$
\bmod \left(\theta_{i k}, \frac{\pi}{2}\right) \neq 0,(k=1,2, \cdots, p)
$$

where $\bmod (\cdot)$ is the remainder operator, then node $v_{i}$ is considered to be an interference node. It should be removed. In Figure 9a, node 2 and node 3 are considered to be interference nodes and removed from the BLG. Finally, the Kruskal algorithm is used to the remaining nodes to generate a new MST which is denoted as $T_{2}$. This refreshed MST shown in Figure 9b represents the final interior layout of the building. Therefore, the MST-based method can also be applied in building interior layout reconstruction even if there exist interference targets.

\section{Conclusions}

We have proposed a building interior layout reconstruction algorithm assuming that complete positions and orientations information of the graph nodes have been obtained. The proposed MST-based method can also deal with the case when there exist interference targets. It is hoped that based on these preliminary results, we are able to predict the building interior layout using radar measurements. However, in practical situation, some trihedral nodes may not be detected in the radar image. Extension of this work is to solve this problem. It is presently under study and the results will be presented in our future research work.

\section{Competing interests}

The authors declare that they have no competing interests.

\section{Acknowledgements}

This work was supported by the National Natural Science Foundation of China under Grants 61271441, 61372161 and the Foundation for the Author of National Excellent Doctoral Dissertation of China under Grant 201046.

Received: 16 December 2013 Accepted: 27 February 2014

Published: 12 March 2014

\section{References}

1. EJ Baranoski, Through wall imaging: historical perspective and future directions, in Proc. IEEE ICASSP, 2008, pp. 5173-5176

2. SE Borek, An overview of through the wall surveillance for homeland security, in Proceedings of the 34th Applied Imagery and Pattern Recognition Workshop (AIPR05), 2005, p. 6

3. JJM de Wit, WL van Rossum, FMA Smits, SAPPHIRE: a novel building mapping radar, in Proceedings of the 39th European Microwave Conference, 2009, pp. 1896-1899

4. JJM de Wit, L Anitori, WL van Rossum, RG Tan, Radar mapping of buildings using sparse reconstruction with an overcomplete dictionary, in Proceedings of the 8th European Radar Conference, 2011, pp. 9-12

5. F Soldovieri, R Solimene, Through-wall imaging via a linear inverse scattering algorithm. IEEE Geosci. Remote Sens. Lett. 4(4), 513-517 (2007)

6. C Le, T Dogaru, L Nguyen, MA Ressler, Ultrawideband (UWB) radar imaging of building interior: measurements and predictions. IEEE Trans. Geosci. Remote Sens. 47(5), 1409-1420 (2009)

7. T Dogaru, A Sullivan, C Kenyon, C Le, Radar signature prediction for sensing-through-the-wall by Xpatch and AFDTD, in DoD High Performance Computing Modernization Program Users Group Conference, 2009, pp. 339-343

8. E Ertin, RL Moses, Through-the-wall SAR attributed scattering center feature estimation. IEEE Trans. Geosci. Remote Sens. 47(5), 1338-1348 (2009)

9. B Chen, T Jin, Z Zhou, B Lu, Estimation of pose angle for trihedral in ultrawideband virtual aperture radar. Prog. Electromagn. Res. $138,307-325$ (2013) 
10. T Jin, J Lou, Z Zhou, Extraction of landmine features using a forward-looking ground penetrating radar with MIMO array. IEEE Trans. Geosci. Remote Sens. 50(10), 4135-4144 (2012)

11. P Chinling Chang, Near zone radar imaging and feature capture of building interiors. Thesis, The Ohio State University, 2008

12. N Subotic, E Keydel, J Burns, A Morgan, K Cooper, B Thelen, B Wilson, W Williams, S McCarty, B Lampe, B Mosher, D Setterdahl, Parametric reconstruction of internal building structures via canonical scattering mechanisms, in Proc. IEEE ICASSP, 2008, pp. 5189-5192

13. T Jin, B Chen, Z Zhou, Image-domain estimation of wall parameters for autofocusing of through-the-wall SAR imagery. IEEE Trans. Geosci. Remote Sens. 51(3), 1836-1843 (2013)

14. EM Lavely, Y Zhang, EH Hill III, Y Lai, P Weichman, A Chapman, Theoretical and experimental study of through-wall microwave tomography inverse problems. J. Frankl. Inst. 345(6), 592-617 (2008)

15. G Hickman, JL Krolik, A graph-theoretic approach to constrained floor plan estimation from radar measurements. IEEE Trans. Signal Process. 57(5), 1877-1888 (2009)

16. RJ Allison, SP Goodwin, RJ Parker, SFP Zwart, R de Grijs, MBN Kouwenhoven, Using the minimum spanning tree to trace mass segregation. Mon. Not. R. Astron. Soc. 395, 1449-1454 (2009)

17. S Sundar, A Singh, A swarm intelligence approach to the quadratic minimum spanning tree problem. Inf. Sci. 180, 3182-3191 (2010)

18. R Campana, E Massaro, D Gasparrini, S Cutini, A Tramacere, A minimal spanning tree algorithm for source detection in $\mathrm{\gamma}$-ray images. Mon. Not. R. Astron. Soc. 383, 1166-1174 (2008)

19. B Chen, T Jin, B Lu, Z Zhou, P Zheng, A graph-theoretic approach to building layout reconstruction from radar measurements, in 2013 International Symposium on Antennas and Propagation (ISAP2013), 2013, pp. 1276-1279

20. X Wang, $G$ Wang, On the least generated tree with restricted conditions. J. Naval. Aeronaut. Eng. Acad. 14(3), 235-236 (1999)

\section{Submit your manuscript to a SpringerOpen ${ }^{\circ}$ journal and benefit from:}

- Convenient online submission

- Rigorous peer review

- Immediate publication on acceptance

- Open access: articles freely available online

- High visibility within the field

- Retaining the copyright to your article

Submit your next manuscript at $>$ springeropen.com 